\title{
CONVERGENCE OF ITERATIVE SOLUTIONS TO INTEGRAL EQUATIONS FOR SOUND RADIATION*
}

\author{
By GEORGE CHERTOCK (Naval Ship Research and Development Center, Washington, D. C.)
}

1. Introduction. In a recent paper [1] it was shown that the sound pressure at the surface of a body vibrating in a specified but arbitrary pattern could be calculated by solution of an integral equation. And once this is done, the entire sound radiation field could be calculated by simple quadratures from the Helmholtz integral. The method of solution can be used for nonanalytical surface shapes and vibration patterns such as occur in technical engineering usage, and is easily adapted to machine calculation.

In such applications, a preferred method for solving the integral equation is by an iterative solution of a finite set of simultaneous equations which approximate the integral equation, because any more direct method may require excessive storage capacity in the computer. This paper proposes and illustrates a more general iteration scheme than the classical Neumann series and derives conditions for its convergence which apply when the kernel function of the integral equation has a spectrum of eigenvalues and a complete set of eigenfunctions defined on the vibrating surface. If there are no eigenvalues the simple Neumann series always converges.

2. Iterative solution to the integral equation. The integral equation for the sound pressure at the vibrating surface is of the form

$$
p(s)=f(S)+\iint K\left(S, S^{\prime}\right) p\left(S^{\prime}\right) d \sigma^{\prime}
$$

where $S$ and $S^{\prime}$ are two points on the surface, $f(S)$ is a known function related to the vibration pattern,

$$
K\left(S, S^{\prime}\right)=\frac{\partial}{\partial n^{\prime}}\left(\frac{e^{i k R}}{2 \pi R}\right)
$$

$k$ is a wavenumber, $R$ is the distance between $S$ and $S^{\prime}, n^{\prime}$ is the normal to the surface at $S^{\prime}$, and the integration is over the surface area. The kernel function here is a particular solution to the scalar wave equation. A sufficient condition that it have a complete set of eigenvalues on the surface is that the surface is one of a family of confocal quadric surfaces [2].

The proposed iteration scheme is of the form

$$
p^{(n+1)}=H p^{(n)}+(1-H)\left(f+\iint K p^{(n)} d \sigma^{\prime}\right)
$$

where $p^{(n)}$ denotes the $n$th iterate and $H$ is a complex constant. Let $\phi_{i}(S)$ be the eigenfunctions of the kernel and $\lambda_{i}$ be the associated eigenvalues, i.e.

*Received November 7, 1966; revised manuscript received April 6, 1967.

'Temporarily at Department of Applied Mathematics and Theoretical Physics, University of Cambridge. 


$$
\lambda_{i} \iint K\left(S, S^{\prime}\right) \phi_{i}\left(S^{\prime}\right) d \sigma^{\prime}=\phi_{i}(S) .
$$

Then expand $f(S)$ and $p^{(n)}(S)$ in terms of these eigenfunctions, and (3) becomes

$$
p^{(n+1)}=\sum \phi_{i}\left[\left(\phi_{i}, f\right)(1-H)+\left(\phi_{i}, p^{(n)}\right)\left(H+\gamma_{i}-H \gamma_{i}\right)\right],
$$

where $\gamma_{i}=\lambda_{i}^{-1}$ and $\left(\phi_{i}, f\right)=\iint \phi_{i} f d \sigma$. Or, by induction,

$$
\begin{aligned}
p^{(n+1)} & =\sum \phi_{i}\left[\left(\phi_{i}, f\right)(1-H)\left(1+A_{i}+A_{i}^{2}+\cdots+A_{i}^{n-1}\right)+\left(\phi_{i}, p^{(1)}\right) A_{i}^{n}\right] \\
& =\sum \phi_{i}\left[\left(\phi_{i}, f\right)(1-H)\left(\left(1-A_{i}^{n}\right) /\left(1-A_{i}\right)\right)+\left(\phi_{i}, p^{(1)}\right) A_{i}^{n}\right]
\end{aligned}
$$

where $A_{i}=\gamma_{i}+H-\gamma_{i} H$. Hence if $\left|A_{i}\right|<1$, then

$$
p^{(n+1)} \rightarrow \sum \phi_{i}\left(\phi_{i}, f\right)(1-H) /\left(1-A_{i}\right)=\sum \phi_{i}\left(\phi_{i}, f\right) /\left(1-\gamma_{i}\right)=p(S)
$$

for all choices of $p^{(1)}$.

Thus the condition that (3) converge to the solution of the integral equation is that all the $A_{i}$ are within the unit circle in the complex plane. A more useful condition which can easily be verified is that the reciprocal eigenvalues $\gamma_{i}=\lambda_{i}^{-1}=\left(H-A_{i}\right) /(H-1)$ are all within the circle passing through the point $(1,0)$, with center at $H /(H-1)$, and with radius equal to $|1 /(1-H)|$.

When $H=0$ (and $p^{(1)}=f$ ) this iteration scheme reduces to a simple Neumann series for which the convergence requirement is that there be no eigenvalues $\lambda_{i}$ within the unit circle. The more general case where $H$ is a real constant was apparently first analysed by Wiarda [3] for symmetric kernels, and later by Buckner [4] for more general kernel functions. If $H$ is a positive number less than unity the domain of convergence is a larger circle including the unit circle of the simple iteration scheme. In fact this condition, $0 \leq H \leq 1$, was discovered and used on an ad hoc basis in the examples described in [1].

The case of complex $H$ is a special case of general iterative methods for operator equations in abstract Hilbert or Banach spaces which have been investigated by Schönberg [5], Rall [6], and Petryshyn [7]. However, it was independently derived as in the present paper before the author was aware of the prior investigations, in order to solve the sound radiation problem discussed below.

3. Numerical example. We consider the surface of a prolate spheroid of reciprocal eccentricity $\xi_{0}=1.10$ which vibrates only in a narrow band between $\eta=0.537$ and $\eta=0.620$ ( $\eta$ is the spheroidal angle coordinate) with velocity $v_{0}$ normal to the longitudinal axis. The vibration frequency is specified by $k a=7.26$ where $k$ is the wave number and $a$ is the semimajor axis. The integral equation for the sound pressure at the surface is

$$
p(\eta)=f(\eta)+\int_{-1}^{+1} K\left(\eta, \eta^{\prime}\right) p\left(\eta^{\prime}\right) d \eta^{\prime}
$$

where the kernel function is now

$$
K\left(\eta, \eta^{\prime}\right)=\int_{-\pi}^{+\pi} \frac{a\left(\xi_{0}^{2}-1\right)}{2 \pi \xi_{0}}\left[\frac{\partial}{\partial \xi^{\prime}}\left(\frac{e^{i k R}}{R}\right)\right]_{\xi_{0}} d \phi^{\prime}
$$

and where $R$ is the distance between two points $(\xi, \eta, \phi)$ and $\left(\xi^{\prime}, \eta^{\prime}, \phi^{\prime}\right)$ (in spheroidal coordinates) on the spheroid $\xi^{\prime}=\xi=\xi_{0}$; and where 


$$
f(\eta)=\frac{i \rho c v_{0} k a^{2}\left(\xi_{0}^{2}-1\right)^{1 / 2}}{-2 \pi \xi_{0}} \int_{-1}^{+1} \int_{-\pi}^{\pi} \frac{e^{i k R}}{R}\left(1-{\eta^{\prime 2}}^{1 / 2} d \eta^{\prime} d \phi^{\prime}\right.
$$

and $\rho$ is the density and $c$ the sound velocity in the fluid.

Then, by expansion of the kernel function in spheroidal wave functions [8], it may be verified that the kernel has eigenfunctions $\phi_{i}(\eta)=S_{0 i}(k a, \eta)$ which are the spheroidal angle functions of the first kind, of order 0 , and degree $i$. The associated eigenvalues $\lambda_{i}$ are given by

$$
\lambda_{i}^{-1}=1-2 k a\left(\xi_{0}^{2}-1\right) R^{(1)} R^{(2)}+2 i k a\left(\xi_{0}^{2}-1\right) R^{(1)} R^{(1)}
$$

where $R_{0 i}^{(1)}(k a, \xi)$ and $R_{0 i}^{(2)}(k a, \xi)$ are the spheroidal radial functions of the first and second kind respectively, and the prime denotes a derivative with respect to $\xi$.

The numerical values for the first seven reciprocal eigenvalues are plotted in Fig. 1. The values for $i>6$ are presumed to cluster about the origin. Four of these $\gamma_{i}$ are outside the unit circle, which means that an iteration with $H=0$ will not converge. When $H=\frac{1}{2}$, or when $H$ is any real number smaller than unity, $\gamma_{1}$ remains outside the circle of convergence. But when $H=(1+i) / 2$, all $\gamma_{i}$ are within the circle and the iteration process of (3) converges for all $f(\eta)$ and for any choice of $p^{(1)}$.

Using this value of $I$, Fqs. (8)-(10) were solved on an IBM 7090 computer with the results for the real and imaginary parts of the surface pressure, $p_{1}$ and $p_{2}$ respectively, shown in Fig. 2.

An explicit, noniterative solution to this particular problem can easily be given as a series in spheroidal wave functions. But this series would be most laborious to evaluate numerically because the functions have not been tabulated. Since the width of the moving zone is only about $1 / 25$ of the length of the spheroid, it would be necessary to use more than 25 terms in the series expansion for the velocity distribution in order to achieve any reasonable accuracy. In fact Hanish [9] did compute the first 20 orders of spheroidal wave functions for this problem and used them to calculate the surface

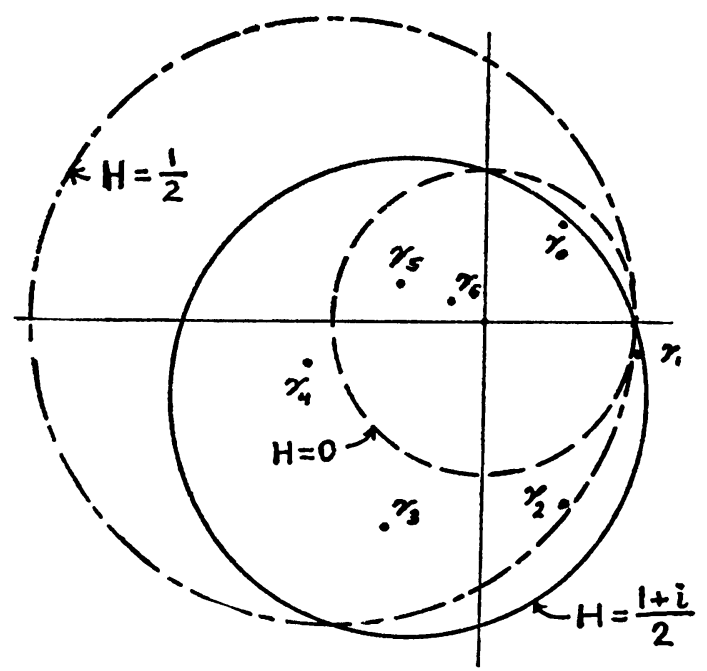

Fia. 1. Location of reciprocal eigenvalues $\gamma_{i}$ relative to circles of convergence for different values of $H$. 


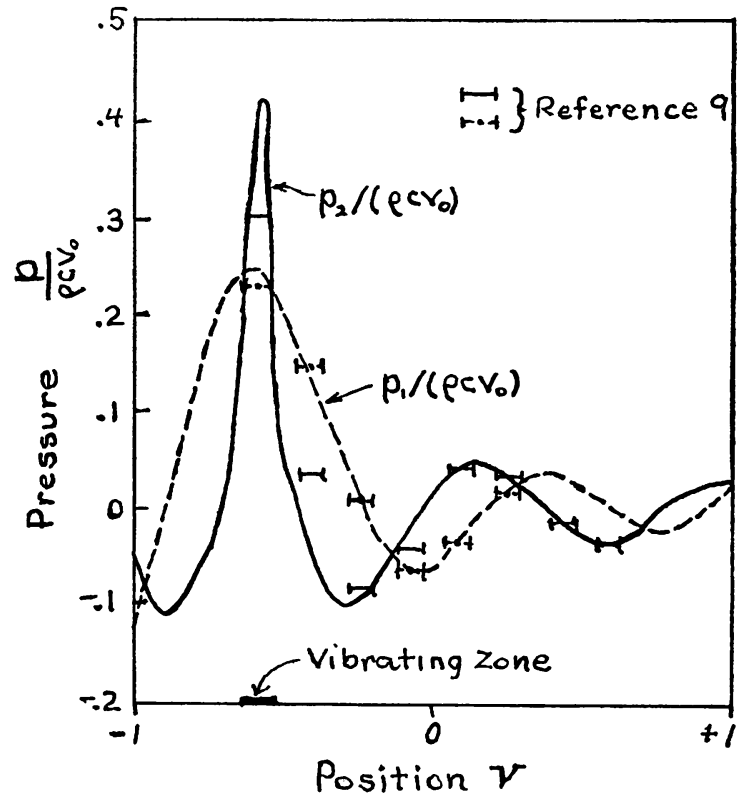

Fig. 2. Sound pressure at surface of spheriod compared with mean values from [9].

pressure. He reported only the mean surface pressure at eight different narrow zones on the surface, each value being averaged over the width of the zone as indicated by the width of the bar in Fig. 2. Note that the real components of his mean values are in fair agreement with the values computed here, but the imaginary component at the moving zone is too low by 25 percent or so.

4. Remarks. An alternative method which is being used to solve sound radiation problems [10], [11] (or more generally to solve the wave equation [12] or the Laplace equation [13] with mixed boundary conditions) requires that the strength of the equivalent simple source surface distribution be determined by solution of a Fredholm integral equation. But the kernel function in this equation is exactly the same as in Eq. (2), and so the problems of convergence of an iterative solution are the same as discussed here.

\section{ReFerences}

[1] G. Chertock, Sound radiation from vibrating surfaces, J. Acoust. Soc. Amer. 36, 1305-1313 (1964)

[2] P. M. Morse and H. Feshbach, Methods of theoretical physics, McGraw-Hill, New York, 1953, Chapter 11

[3] G. Wiarda, Integralgleichungen unter besonderen Berucksichtigung der Anwendungen, Leipzig, 1930

[4] H. Buckner, A special method of successive approximations for Fredholm integral equations, Duke Math. J. 15, 197-206 (1948)

[5] M. Schönberg, Sur la methode d'iteration de Wiarda et de Buckner pour la resolution de Fredholm, Acad. Roy. Belgique, Bull. Cl. Sci. 37, 1941-1156 (1951); 38 and 154-167 (1952)

[6] L. B. Rall, Error bounds for iterative solutions to integral equations, Pacific J. Math. 5, 977-986 (1955)

[7] W. V. Petryshyn, On a general iterative method for the approximate solution of linear operator equation, Math Comp. 17, 1-10 (1963)

[8] C. Flammer, Spheroidal wave functions, Stanford University Press, Stanford, Calif., 1957, p. 47

[9] S. Hanish, The numerical computation of radiation impedance of zonal array on a hard prolate spheroidal baffle, Naval Research Laboratory Report 6108, July 1964 
[10] L. H. Chen and D. G. Schweikert, Sound radiation from an arbitrary body, J. Acoust. Soc. Amer. 35, (1963)

[11] R. P. Banaugh and W. Goldsmith, Diffraction of steady acoustic waves by surfaces of arbitrary shape, J. Acoust. Soc. Amer. 35, 1590-1601 (1963)

[12] G. B. Brundrit, $A$ solution to the problem of scalar scattering from a smooth bounded surface using integral equations, Quart. J. Mech. Appl. Math. 18, 473-489 (1965)

[13] J. L. Hess and A. M. O. Smith, Calculation of non-lifting potential flow about arbitrary bodies, Douglas Aircraft Co. Report ES 4062, March 1962 